VOL. 46 (1992) [1-21]

\title{
A NOTE ON COVERINGS AND KERVAIRE COMPLEXES
}

\author{
STEPHEN G. BRICK
}

In the combinatorial category, a two-complex $X$ is said to be Kervaire if any set of equations modelled on $X$, over any group, has a solution in a larger group. The Kervaire-Laudenbach conjecture speculates that if $\mathrm{H}_{2}(X)=0$ then $X$ is Kervaire. We show that the validity of this conjecture would imply that all aspherical twocomplexes are Kervaire. In particular, any two-complex homotopically equivalent to a two-manifold $\left(\neq S^{2}, R P^{2}\right)$ would be Kervaire. We show that this is indeed the case for certain such two-complexes. We generalise this to staggered twocomplexes, and, more generally, one-relator extensions of Kervaire complexes. We obtain similar results for diagrammatically reducible two-complexes. Our proofs make use of covering spaces.

\section{INTRODUCTION}

The classical Kervaire-Laudenbach conjecture speculates that the trivial group cannot be obtained by starting with a non-trivial group and adding one new generator and one new relator. This conjecture has evolved into one concerning the problem of solving equations over groups.

We recall some terminology (see [5]). An equation over a group $G$ is of the form

$$
w\left(T_{1}, T_{2}, \ldots, T_{n}\right)=1 \text { with } w\left(T_{1}, T_{2}, \ldots, T_{n}\right) \in G * F\left(T_{1}, T_{2}, \ldots, T_{n}\right)
$$

where $F\left(T_{1}, T_{2}, \ldots, T_{n}\right)$ is the free group on the unknowns $T_{1}, T_{2}, \ldots, T_{n}$. We say that the equation $w\left(T_{1}, T_{2}, \ldots, T_{n}\right)=1$ can be solved over $G$ if there exists a group $H$ containing $G$ and possessing elements $t_{1}, t_{2}, \ldots, t_{n}$ with $w\left(t_{1}, t_{2}, \ldots, t_{n}\right)=1$ in $H$. This is equivalent to the natural map

$$
G \rightarrow G * F\left(T_{1}, T_{2}, \ldots, T_{n}\right) / \ll w\left(T_{1}, T_{2}, \ldots, T_{n}\right) \gg
$$

being injective. We will refer to the group

$$
G * F\left(T_{1}, T_{2}, \ldots, T_{n}\right) / \ll w\left(T_{1}, T_{2}, \ldots, T_{n}\right) \gg
$$

as the universal solution group of the equation $w\left(T_{1}, T_{2}, \ldots, T_{n}\right)=1$. Similarily we could speak of a set of equations over a group $\mathrm{G}$.

Received 21 June 1991

Copyright Clearance Centre, Inc. Serial-fee code: 0004-9729/92 \$A2.00+0.00. 
Given a set of equations $\left\{w_{i}\left(T_{1}, T_{2}, \ldots, T_{n}\right)=1\right\}$ over a group $\mathrm{G}$, one can form its associated two complex $Y$ as follows:

(1) $Y$ has a single 0 -cell.

(2) $Y$ has a one-cell for each unknown $T_{j}$.

(3) $Y$ has a two-cell for each equation $w_{i}\left(T_{1}, T_{2}, \ldots, T_{n}\right)=1$ with the attaching map obtained from the word $w_{i}$ by deleting occurences of elements of $\mathrm{G}$ - but without reducing the word in the unknowns so obtained.

We say that the set of equations $\left\{w_{i}=1\right\}$ is modelled on the complex $Y$. In general, if $X$ is a two-complex, a set of equations is said to be modelled on $X$ if the associated two-complex is $X / X^{(0)}$.

There is another way of viewing which equations are modelled on a two-complex $X$. Fix a group $G$ and an orientation for each two-cell of $X$. For each (oriented) two-cell, choose some group element for each "corner" of the two-cell. Reading around each two-cell, yields an equation over $G$, where the unknowns are the one-cells of $X$.

Observe that the two-complex associated to a set of equations over a group is necessarily a combinatorial complex. (Recall that a cellular map is combinatorial if it preserves dimension on each open cell and a complex is combinatorial if its attaching maps, after a possible subdivision, are combinatorial.) With this in mind we say that a combinatorial two-complex $X$ is Kervaire if any set of equations modelled on $X$ (over any group) can be solved.

It works out that the question of whether a two-complex is Kervaire does not depend on the combinatorial structure, that is, it is a topological property (see [1]). Some examples of Kervaire complexes are all surfaces other than the two-sphere, any collapsible two-complex, and the Dunce Hat (see $[1,3,7]$ ).

Topologically we can rephrase the definition of a Kervaire complex. In order to do so we need to define the quotient in the category of combinatorial two-complexes (also see [3]).

Let $K \subset L$ be a pair of combinatorial two-complexes. Their quotient, $L / / K$, will be defined provided that no two-cell of $L \backslash K$ has the image of its attaching map contained entirely in $K$. With this condition satisfied, the combinatorial quotient $L / / K$ has one skeleton being the standard quotient $L^{(1)} / K^{(1)}$. For each two-cell $\Delta$ of $L \backslash K$, there is a corresponding two-cell $\Delta^{\prime}$ of $L / / K$. We need only specify the attaching map of $\Delta^{\prime}$. Let $\alpha: \partial \Delta \rightarrow L^{(1)}$ be the attaching map of $\Delta$. Collapse each component of $\alpha^{-1}\left(K^{(1)}\right)$ to a point. The resulting quotient space of the complex $\partial \Delta$ is a circle with an induced cell structure. Identify it with $\partial \Delta^{\prime}$ and let the map induced by $\alpha$, say $\alpha^{\prime}: \partial \Delta^{\prime} \rightarrow L^{(1)} / K^{(1)}$, be the attaching map for $\Delta^{\prime}$. Clearly, $\alpha^{\prime}$ is a combinatorial map. Also note that $L / / K$ is a quotient space of $L / K$ and the quotient map is a homotopy equivalence. 
Using this combinatorial quotient, we have the following definition of the Kervaire property:

Definition: Let $X$ be a combinatorial two-complex. $X$ is said to be Kervaire if given any pair of combinatorial two-complexes $K \subset L$ with $L / / K=X / X^{(0)}$ the natural map $\pi_{1}(K) \rightarrow \pi_{1}(L)$ is injective.

It is not difficult to see that this definition agrees with the one above. It is implicit, for instance, in the proof of Proposition 3.2 of [1]. Also see [7]. But it is quite simple to see the equivalence of the definitions directly. To that end, let $\left\{w_{i}\left(T_{1}, T_{2}, \ldots, T_{n}\right)=1\right\}$ be a set of equations over a group $G$. Construct a two-complex $K$ with fundamental group $G$ with a single vertex. Now adjoin a one-cell for each unknown $T_{j}$. For each equation $w_{i}=1$ adjoin a two-cell whose attaching map gives the word $w_{i}\left(T_{1}, T_{2}, \ldots, T_{n}\right) \in G * F\left(T_{1}, T_{2}, \ldots, T_{n}\right)$. Call the resulting two-complex $L$. By Van-Kampen's Theorem, the natural map

$$
\pi_{1}(K) \rightarrow \pi_{1}(L)
$$

is the same as the map

$$
G \rightarrow G * F\left(T_{1}, T_{2}, \ldots, T_{n}\right) / \ll\left\{w_{i}\left(T_{1}, T_{2}, \ldots, T_{n}\right)\right\} \gg
$$

Also, one has that $L / / K=X / X^{(0)}$. On the other hand, if one has a pair of combinatorial two-complexes $K \subset L$ where $L^{(0)}=K^{(0)}$ then, applying Van-Kampen's Theorem, one has a set of equations modelled on $L / / K$ over the group $\pi_{1}(K)$ with $\pi_{1}(L)$ being the universal solution group.

Using this topological formulation, the Kervaire conjecture (also known as the Kervaire-Laudenbach conjecture) becomes the following :

Conjecture. Let $X$ be a combinatorial two-complex. Assume that $H_{2}(X)=0$. Then $X$ is Kervaire.

We give a slightly different formulation of the Kervaire property due to Gersten (unpublished). We show below that the two definitions are in fact equivalent.

Our aim is to give a method that yields more examples of Kervaire complexes. The key idea is to use the following result:

Theorem 2.2. If $\tilde{X} \rightarrow X$ is a covering space and $\tilde{X}$ is Kervaire then $X$ is Kervaire.

A simple application of the preceding theorem yields:

COROLlary 2.3. If the Kervaire conjecture is true then all aspherical combinatorial two-complexes are Kervaire. 
In particular, any two-complex homotopy equivalent to a surface $\left(\neq S^{2}, R P^{2}\right)$ should be Kervaire. Thus, for example, the two-complex

$$
K=\mathcal{K}\left\langle x, y \mid x^{2} x^{-1} y^{n+1} y^{-n} x^{k} x^{-k-1} y^{-m-1} y^{m}\right\rangle
$$

where $n, k$, and $m$ are integers should be Kervaire. We show that this is indeed the case. Our approach is to study the universal cover $\widetilde{K}$ of $K$.

More generally we show that a one-relator extension, by a non-power, of a Kervaire complex, whose fundamental group is locally indicable, is Kervaire (see Section 4 below for the definitions). Our method of proof is to analyze the universal cover of the extended complex, applying results of Howie (see [6] and below).

We also obtain similar results for diagramatically reducible two-complexes (defined in Section 2, below). We end the paper with a conjecture on the structure of diagramatically reducible two-complexes.

A few of these results were also obtained independently by Gersten (unpublished).

We make use of polyhedral constructions - such as Link and Star - which implicitly takes place in the second barycentric subdivision. Further, we use "cl" to denote closure.

\section{Preliminary Results}

We need to recall a few notions (see [1]).

Let $(B, A)$ be a pair of topological spaces and let $f:(F, \partial F) \rightarrow(B, A)$ be a map of a disk with holes. We may designate a component of $\partial F$ as the outer boundary component. When such a choice has been made, we call $f$ a based map. Suppose we are given two based maps $f:(F, \partial F) \rightarrow(B, A)$ and $\widehat{f}:(\widehat{F}, \partial \widehat{F}) \rightarrow(B, A)$ of disks with holes $F$ and $\widehat{F}$. We say that the map $\widehat{f}$ has boundary labels derived from $f$ if the following holds:

Let $a_{0}, a_{1}, \ldots, a_{n}$ and $\widehat{a}_{0}, \widehat{a}_{1}, \ldots, \widehat{a}_{m}$ be the boundary components of $F$ and $\widehat{F}$ respectively, where $a_{0}$ and $\widehat{a}_{0}$ are the outer boundary components. Then there is a mapping of indices $\beta:\{0,1, \ldots, m\} \rightarrow\{0,1, \ldots, n\}$ with $\beta(0)=0$ and $\beta(i)>0$ for each $i>0$. And for each $i$ there is a homeomorphism $\beta_{i}: \widehat{a}_{i} \rightarrow a_{\beta(i)}$ with $\widehat{f} \mid \hat{a}_{i}=f \circ \beta_{i}$.

It should be noted that no relationship between $n$ and $m$ is assumed. Furthermore, the map of indices $\beta$ need not be either injective or surjective. Note that in [1] we only require $\hat{f} \mid \widehat{a}_{i}=f \circ \beta_{i}$ up to homotopy. As the homotopy is one of maps into $A$, we lose nothing with the stronger requirement of equality. Also, we will typically require that $\widehat{f}(\widehat{F}) \subset A$.

Let $X$ be a combinatorial two-complex. A spherical diagram over $X$ is a combinatorial map $\phi: T \rightarrow X$ where $T$ is a combinatorial tesselation of the twosphere. Set $L_{X}=\operatorname{Link}\left(X^{(0)}, X\right), F=\operatorname{cl}\left(T \backslash N_{T}\right)$ and $X_{1}=\operatorname{cl}\left(X \backslash N_{X}\right)$ where 
$N_{T}=\operatorname{Star}\left(T^{(0)}, T\right)$ and $N_{X}=\operatorname{Star}\left(X^{(0)}, X\right)$ are the polyhedral regular neighbourhoods of $T^{(0)}$ in $T$ and of $X^{(0)}$ in $X$. Observe that $F$ is a disk with holes. Restricting $\phi$ yields a map $f_{\phi}:(F, \partial F) \rightarrow\left(X_{1}, L_{X}\right)$. We say that $\phi$ has redundant links if for each choice of an outer boundary component there is a based map of a disk with holes $f: \widehat{F} \rightarrow L_{X}$ with boundary labels of $f$ derived from $f_{\phi}$ (note - the point is that $f$ has image contained entirely in the subcomplex $L_{X}$ ).

We then have the following characterisation of Kervaire complexes:

Proposition 1.1. Let $X$ be a combinatorial two-complex. Then $X$ is Kervaire if and only if every spherical diagram over $X$ has redundant links.

Proof: See [1, Proposition 3.2].

We now state the formulation of the Kervaire property that Gersten uses (unpublished).

Definition: Let $X$ be a combinatorial two-complex. $X$ is said to be G-Kervaire if given any pair of combinatorial two-complexes $A \subset B$ with $B / / A=X$ the natural map $\pi_{1}(A) \rightarrow \pi_{1}(B)$ is injective.

Observe that $A$ can be thought of as a vertex of $X$. For different choices of $A$ and $B$, the vertex of $X$ may be different. And since we quantify over all pairs $A$ and $B$ with $B / / A=X$, each vertex of $X$ occurs as some $A$. We now address the equivalence of the G-Kervaire and the Kervaire properties.

Proposition 1.2. Let $X$ be a combinatorial two-complex. Then $X$ is Kervaire if and only if $X$ is $G$-Kervaire.

Proof: $\Rightarrow$ : Assume $X$ is Kervaire and $A \subset B$ are combinatorial two-complexes with $B / / A=X$. Let $L=B / B^{(0)}$ and $K=A / A^{(0)}$. Then $K \subset L$ and $L / / K=$ $X / X^{(0)}$. Let $i: K \subset L$ be the inclusion and let $v$ be the unique 0 -cell of $K$. Since $X$ is Kervaire, we have that $\pi_{1}(K, v) \rightarrow \pi_{1}(L, v)$ is injective. Now let $u$ be a 0 -cell of $A$. It is easy to see that $\pi_{1}(K, v)=\pi_{1}(A, u) * F_{A}$ where $F_{A}$ is free with basis $A^{(0)} \backslash\{u\}$. Similarily, we have $\pi_{1}(L, v)=\pi_{1}(B, u) * F_{B}$, where $F_{B}$ is free with basis $B^{(0)} \backslash\{u\}$. Moreover, letting $j: A \subset B$ be the inclusion, we have, under the above identifications, $i_{*} \mid \pi_{1}(A, u)=k \circ j_{*}$ where $k: \pi_{1}(B, u) \subset \pi_{1}(B, u) * F_{B}$ is the inclusion. So in particular, as $i_{*}$ is injective, $j_{*}: \pi_{1}(A, u) \rightarrow \pi_{1}(B, u)$ is injective. Thus it follows that $X$ is G-Kervaire.

$\Leftarrow$ : Suppose that $X$ is G-Kervaire and $\phi: T \rightarrow X$ is a spherical diagram over $X$. We need to see that $\phi$ has redundant links. As above set $N_{T}=\operatorname{Star}\left(T^{(0)}, T\right)$, $N_{X}=\operatorname{Star}\left(X^{(0)}, X\right), L_{X}=\operatorname{Link}\left(X^{(0)}, X\right), F=\operatorname{cl}\left(T \backslash N_{T}\right), X_{1}=\operatorname{cl}\left(X \backslash N_{X}\right)$, and $f_{\phi}:(F, \partial F) \rightarrow\left(X_{1}, L_{X}\right)$ the restriction of $\phi$. Choose an outer boundary component $a_{0}$ of $\partial F$, letting $a_{1}, a_{2}, \ldots, a_{n}$ be the remaining boundary components. We will show that, for this choice of an outer boundary component, there is a based map of a disk 
with holes whose boundary labels are derived from $f_{\phi}$. By Proposition 1.1, because the choice of $a_{0}$ was arbitrary, this will suffice.

Let the vertices of $T$ corresponding to $a_{0}, a_{1}, \ldots, a_{n}$ be $v_{0}, v_{1}, v_{2}, \ldots, v_{n}$. Assume that the vertices (and boundary components) have been ordered so that there is a $k$ with $0 \leqslant k \leqslant n$ such that $\phi\left(v_{i}\right)=\phi\left(v_{0}\right)$ if and only if $i \leqslant k$. Thus the boundary components $a_{0}, \ldots, a_{k}$ all map to $\operatorname{Link}\left(\phi\left(v_{0}\right), X\right)$ while the boundary components $a_{k+1}, \ldots, a_{n} \operatorname{map}$ to the links of other vertices of $X$. We now construct a pair of two-complexes $A \subset B$ as follows. Let $A_{0}=\operatorname{Link}\left(\phi\left(v_{0}\right), X\right)$. For each $1 \leqslant i \leqslant k$ attach a two-cell to $A_{0}$ using the map $f_{\phi}\left\lceil a_{i}\right.$ as the attaching map (so if $k=0$, nothing is attached). Call the resulting two-complex $A$. The complex $B$ is defined to be $\operatorname{cl}\left(X \backslash \operatorname{star}\left(\phi\left(v_{0}\right), X\right)\right) \cup A$.

Since $\operatorname{Link}\left(\phi\left(v_{0}\right), X\right) \subset A$ and $\operatorname{cl}\left(X \backslash \operatorname{star}\left(\phi\left(v_{0}\right), X\right)\right) / / \operatorname{Link}\left(\phi\left(v_{0}\right), X\right)=X$, it follows that $B / / A=X$ and so, since $X$ is assumed to be G-Kervaire, the natural map $\pi_{1}(A) \rightarrow \pi_{1}(B)$ is injective.

Now $\operatorname{cl}\left(T \backslash \operatorname{star}\left(v_{0}, T\right)\right)$ is a disk $D$ with boundary being $a_{0}$. By restriction and by the construction of the pair $(B, A), \phi$ induces a map $\phi^{\prime}:(D, \partial D) \rightarrow(B, A)$. Since $\pi_{1}(A) \rightarrow \pi_{1}(B)$ is injective, $\left[\phi^{\prime}\left\lceil a_{0}\right]=1\right.$ in $\pi_{1}(A)$. So there is a map of a disk $h: \widehat{D} \rightarrow A$ with $\partial \widehat{D}$ mapping according to $\phi^{\prime}\left\lceil a_{0}\right.$. Assume that $h$ is transverse to the cell structure of $A$. Let

$$
\left.\widehat{F}=\mathrm{cl}\left(\widehat{D} \backslash h^{-1} \text { (the interior of the two cells of } A\right)\right)
$$

and let $f$ be the restriction of $h$. Take as outer boundary component of $\widehat{F}$ the boundary component of $\hat{D}$. Then $f$ is a based map of a disk with holes. Moreover

$$
f\left\lceil(\text { the outer boundary component })=h\left\lceil\partial D=\phi^{\prime}\left\lceil a_{0}=f_{\phi}\left\lceil a_{0}\right.\right.\right.\right.
$$

And

$f\left\lceil(\right.$ an inner boundary component $)=(\text { the attaching map of some two cell of } A)^{ \pm 1}$

But these two-cells were attached according to map $f_{\phi} \mid a_{i}$ for some $i$ with $1 \leqslant i \leqslant k$. It follows that $f$ has boundary labels derived from $f_{\phi}$.

By making use of the G-Kervaire formulation one can obtain a local characterisation of Kervaire complexes. We start by recalling a definition. Suppose $(B, A)$ is a pair of topological spaces. We say that $A$ is normal-convex in $B$ if given any based map $f:(F, \partial F) \rightarrow(B, A)$ of a disk with holes there exists a based map of another disk with holes $\widehat{f}: \widehat{F} \rightarrow A$ with boundary labels derived from $f$. In [1] it is shown that $X$ is Kervaire iff the graph $\operatorname{Link}\left(X^{(0)}, X\right)$ is normal-convex in $\operatorname{cl}\left(X \backslash \operatorname{star}\left(X^{(0)}, X\right)\right)$. We modify the argument there to the case of G-Kervaire to obtain the following. 
Proposition 1.3. Let $X$ be a combinatorial two-complex. Then $X$ is $G$ Kervaire if and only if for every vertex $v$ of $X$ the graph $\operatorname{Link}(v, X)$ is normal-convex in $\operatorname{cl}(X \backslash \operatorname{Star}(v, X))$.

Proof: $\Leftrightarrow:$ We prove the contrapositive. Assume that $X$ is not G-Kervaire. Let $A \subset B$ be combinatorial two-complexes with $B / / A=X$ but with the natural map $\pi_{1}(A) \rightarrow \pi_{1}(B)$ not being injective. Denote by $v$ the vertex of $X$ corresponding to $A$. We will show that the graph $L_{v}=\operatorname{Link}(v, X)$ is not normal-convex in $X_{v}=$ $\operatorname{cl}(X \backslash \operatorname{Star}(v, X))$.

As $\pi_{1}(A) \rightarrow \pi_{1}(B)$ is not injective, we can find a map of a disc $f: D^{2} \rightarrow B$ with $f\left\lceil\partial D^{2}: \partial D^{2} \rightarrow A\right.$ being an essential loop in $A$. Assume that $f$ is transverse to the cell-structure of $B$, and consider the composite map $f_{1}=p \circ f: D^{2} \rightarrow B \rightarrow B / A \rightarrow$ $B / / A$ where $p: B \rightarrow B / A \rightarrow B / / A$ is the composite of the natural quotient maps (Recall that $B / / A$ is a quotient of $B / A$ ). We then have a tamely-embedded graph $\Gamma \subset D^{2}$ with $f_{1}$ mapping regular neighbourhoods of the vertices homeomorphically onto two-cells of $X$, mapping arcs transverse to the edges of $\Gamma$ onto one-cells of $X$, and mapping the complement of a regular neighbourhood $N(\Gamma)$ of $\Gamma$ to zero-cells of $X$. Recall (see [1]) that this graph $\Gamma$, together with how $f_{1}$ maps a regular neighbourhood of it, is called the picture of $f_{1}$.

Since $f\left(\partial D^{2}\right) \subset A$ and $A$ is collapsed to a vertex of $X$, we have that $\Gamma \subset \operatorname{int}\left(D^{2}\right)$. If $\Gamma$ is not connected then for some simple closed curve $C \subset f_{1}^{-1}$ (the vertex A) there are components of $\Gamma$ both in the inside and the outside of $C$ in $D^{2}$. If $f(C)$, a loop in $A$, is essential in $A$, replace $D^{2}$ by the subdisc $\Delta$ interior to $C$ and $f$ by $f \mid \Delta$. Otherwise, alter $f$ in $\Delta$ so that $f(\Delta) \subset A$. In this way we reduce the number of components of $\Gamma$ without losing the property that $f\left\lceil\partial D^{2}\right.$ is essential in- $A$. Hence we may assume that $\Gamma$ is connected.

Since $\Gamma$ is connected, $N=N(\Gamma)$ is a disk with holes. We want to look at maps of disks with holes into $\left(X_{v}, L_{v}\right)$, but $f_{1}\lceil N$ maps $\partial N$ to the vertex $v$ which is not contained in $L_{v}$. So we need to look at a slightly smaller regular neighbourhood of $\Gamma$ which maps under $f_{1}$ into $X_{v}$ and whose boundary maps to $L_{v}$. Let $F=\left(f_{1}\right)^{-1}\left(X_{v}\right)$. By the construction of $\Gamma$ this has the desired property. $F$ also inherits from $D^{2}$ a choice of an outer boundary component. Thus $f_{1} \mid F:(F, \partial F) \rightarrow\left(X_{v}, L_{v}\right)$ is a based map of a disk with holes.

We claim that there is no based map of a disk with holes with image contained entirely in $L_{v}$ and with boundary labels derived from $f_{1} \mid F$. Once we show this, we can conclude that $L_{v}$ is not normal-convex in $X_{v}$.

So suppose to the contrary that there is a based map of a disk with holes $\widehat{f}_{1}: \widehat{F} \rightarrow$ $L_{v}$ with boundary labels derived from $f_{1} \mid F$. We will derive a contradiction to the assumption that $f \backslash \partial D^{2}$ is essential in $A$. To do so we will make use of two facts. 
First let $\widehat{A}=p^{-1}(\operatorname{Star}(v, X))$. Observe that $\widehat{A}$ is a polyhedral regular neighbourhood of $A$ in $B$, and so it collapses onto $A$. In particular $A$ is a deformation retract of $\widehat{A}$. Second take $L_{A}=\operatorname{Link}(A, B)=p^{-1}\left(L_{v}\right)$. It is easy to see that $p: L_{A} \rightarrow L_{v}$ is a homeomorphism. Let $q: L_{v} \rightarrow L_{A}$ be its inverse.

Consider the map $\widehat{f}=q \circ \widehat{f}_{1}: \widehat{F} \rightarrow L_{v} \rightarrow L_{A}$. Enumerate the boundary components of $F$, and $\widehat{F}$, respectively, by $a_{0}, a_{1}, \ldots, a_{n}$ and $\widehat{a}_{0}, \widehat{a}_{1}, \ldots, \widehat{a}_{m}$ where $a_{0}$ and $\widehat{a}_{0}$ are the outer components. Let $\beta$ be the map of indices, and $\beta_{i}: \widehat{a}_{i} \rightarrow a_{\beta(i)}$ be the homeomorphisms arising from the fact that $\hat{f}_{1}$ has boundary labels derived from $f_{1}$.

Now $a_{0}$ and $\partial D^{2}$ cobound an annulus in $D^{2} \backslash F$. Call this annulus $S$. Also, for each $i \in\{1,2, \ldots, m\}$ let $\Delta_{i}$ be the subdisk of $D^{2}$ that $a_{\beta(i)}$ bounds. Glue the annulus $S$ and the subdisks $\Delta_{i}$, for $i \in\{1,2, \ldots, m\}$, onto the disk with holes $\widehat{F}$ using the homeomorphisms $\beta_{i}$ as the glueing maps. The resulting space is a disk $\widetilde{D}^{2}$. We now define a map $\tilde{f}: \widetilde{D}^{2} \rightarrow \widehat{A}$ by $\tilde{f}=f$ on $S$ and $\Delta_{i}$ while $\tilde{f}=\widehat{f}$ on $\widehat{F}$. This shows that $f\left|\partial \widetilde{D}^{2}=f\right| \partial D^{2}$ is null-homotopic in $\widehat{A}$. And, as $A$ is a deformation retract of $\widehat{A}$, we can conclude that $f \mid \partial D^{2}$ is null-homotopic in $A$. This is the desired contradiction.

$\Rightarrow$ : Suppose that, for some vertex $v$, the graph $L_{v}=\operatorname{Link}(v, X)$ is not normalconvex in $X_{v}=\operatorname{cl}(X \backslash \operatorname{Star}(v, X))$. We will construct combinatorial two-complexes $A \subset B$ with $B / / A=X$ but with the natural map $\pi_{1}(A) \rightarrow \pi_{1}(B)$ not being injective.

By assumption, there is a based map $f:(F, \partial F) \rightarrow\left(X_{v}, L_{v}\right)$ of a disk with holes such that there is no map $\widehat{f}: \widehat{F} \rightarrow L_{v}$ of a disk with holes, with boundary labels derived from $f$. Let the boundary components of $F$ be $a_{0}, a_{1}, a_{2}, \ldots, a_{n}$ where $a_{0}$ is the outer boundary component.

Construct $A$ by attaching $n$ two-cells to $L_{v}$ using the maps $f_{a_{i}}$, for $i=1,2, \ldots, n$, as attaching maps. Let $B=X_{v} \cup A$. Then, clearly, $f$ can be extended to a map of a disk, $\bar{f}:\left(D^{2}, \partial D^{2}\right) \rightarrow(B, A)$, by mapping subdisks that $a_{i}$, for $i \geqslant 1$, now bound to the corresponding attached two-cell of $A$. Moreover, $f\left\lceil\partial D^{2}\right.$ is an essential loop in $A$. For otherwise we could find a map of a disk into $A$, and then using transversality we could find a map of a disk with holes into $L_{v}$ with boundary labels derived from $f$. And this would contradict our choice of $f$.

Now applying Proposition 1.2 to the above, yields a local characterisation of Kervaire complexes.

Corollary 1.4. Let $X$ be a combinatorial two-complex. Then $X$ is Kervaire if and only if for every vertex $v$ of $X$ the graph $\operatorname{Link}(v, X)$ is normal-convex in $c l(X \backslash \operatorname{Star}(v, X))$.

Since the Kervaire property is independent of cell-structure (see [1]), the above yields the following: 
Corollary 1.5. Let $X$ be a combinatorial two-complex. The following are equivalent:
(1) $X$ is Kervaire
(2) $\forall x \in X$ the graph $\operatorname{Link}(x, X)$ is normal-convex in $\operatorname{cl}(X \backslash \operatorname{Star}(x, X))$.

\section{Coverings}

Given a combinatorial two-complex $A$, we can consider $\operatorname{Link}\left(A^{(0)}, A\right)$ either a complex or a topological space. In either case, a combinatorial map $\alpha: A \rightarrow B$ induces a map $\alpha: \operatorname{Link}\left(A^{(0)}, A\right) \rightarrow \operatorname{Link}\left(B^{(0)}, B\right)$. Thus we can view Link as a functor. Similarly, the polyhedral regular neighbourhood (which we've denoted by $N_{T}$ or $N_{X}$ ) is a functor. With this in mind, it is a simple matter to obtain the following:

Lemma 2.1. Let $\phi: T \rightarrow X$ be a spherical diagram over a combinatorial twocomplex. Suppose $\phi$ factors as $\beta \circ \alpha$ where $\alpha: T \rightarrow Y$ and $\beta: Y \rightarrow X$ are combinatorial maps. If $\alpha$ has redundant links then $\phi$ has redundant links.

Proof: Let $N_{T}, N_{Y}$, and $N_{X}$ be the polyhedral regular neighbourhoods of the zero skeletons of $T, Y$, and $X$ respectively. Set $F=\operatorname{cl}\left(T \backslash N_{T}\right), Y_{1}=\operatorname{cl}\left(Y \backslash N_{Y}\right)$, and $X_{1}=\operatorname{cl}\left(X \backslash N_{X}\right)$. Restricting $\phi$ yields a map $f_{\phi}:(F, \partial F) \rightarrow\left(X_{1}, L_{X}\right)$. Likewise, restricting $\alpha$ and $\beta$ yields maps $f_{\alpha}:(F, \partial F) \rightarrow\left(Y_{1}, L_{Y}\right)$ and $f_{\beta}:\left(Y_{1}, L_{Y}\right) \rightarrow\left(X_{1}, L_{X}\right)$. By the functorality observed above, we have that $f_{\phi}$ factors as $f_{\beta} \circ f_{\alpha}$. Assume a choice of an outer boundary component of $F$ has been made. Now $\alpha: T \rightarrow Y$ has redundant links. Thus, there is a based map of a disk with holes $f: \widehat{F} \rightarrow L_{Y}$ with boundary labels of $f$ derived from $f_{\alpha}$. But then $f_{\beta} \circ f: \widehat{F} \rightarrow L_{X}$ is a based map of a disk with holes, and, as is easily seen, has boundary labels derived from $f_{\phi}=f_{\beta} \circ f_{\alpha}$. Since the choice of an outer boundary component was arbitrary, it follows that $\phi$ has redundant links.

We now use our lemma and the lifting property of covering spaces to prove our result about coverings and Kervaire complexes:

Proposition 2.2. If $p: \tilde{X} \rightarrow X$ is a covering space and $\tilde{X}$ is Kervaire then $X$ is Kervaire.

Proof: Suppose $\phi: T \rightarrow X$ is a spherical diagram over $X$. Then, since $S^{2}$ is simply connected, $\phi$ lifts to a combinatorial map $\alpha: T \rightarrow \tilde{X}$, ie $\phi$ factors as $p \circ \alpha$. Now $\tilde{X}$ is Kervaire. Hence $\alpha: T \rightarrow \tilde{X}$ has redundant links. It follows from the previous lemma that $\phi$ has redundant links. Therefore, by Proposition 1.1, $X$ is Kervaire.

Note that the converse is not necessarily true. For example $R P^{2}$ is Kervaire but its universal covering space $S^{2}$ is not.

Since the universal cover of an aspherical complex has second integral homology 
group being zero (by the Hurewicz theorem) we have the following consequence to the above result:

Corollary 2.3. If the Kervaire conjecture is true then all aspherical combinatorial two-complexes are Kervaire.

We now turn to diagramatically reducible ( $D R$ ) two-complexes. Let $f: T \rightarrow X$ be a spherical diagram over a combinatorial two-complex. We say that two distinct faces (ie closed two-cells) $\Delta_{1}$ and $\Delta_{2}$ of $T$ are opposite with respect to $f$ if $\partial \Delta_{1} \cap \partial \Delta_{2} \neq \emptyset$, and if there is an orientation reversing homeomorphism $\alpha: \Delta_{1} \rightarrow \Delta_{2}$ fixing $\Delta_{1} \cap \Delta_{2}$ pointwise with $f \circ \alpha=f\left\lceil\Delta_{1}\right.$. Given such a pair of opposite faces, one can cut them out and identify points on the boundary. This results in a finite number of two-spheres joined together at certain vertices where the total number of faces has been reduced by two. A combinatorial two-complex $X$ is DR if every spherical diagram over $X$ has a pair of opposite faces. In [3] Gersten proves that a two-complex possessing a hyperbolic weight function is DR, and in particular any surface $\left(\not S^{2}, R P^{2}\right)$ is DR. Furthermore he shows that diagramatically reducible two-complexes satisfy the Reciprocity Law, a condition stronger than being Kervaire.

We will need to make use of two simple facts about DR complexes. First, a subcomplex of a DR complex is DR. This is true as any spherical diagram over a subcomplex can be viewed as a spherical diagram over the larger complex. Second, a disjoint union of DR complexes is DR. This follows as the image of a spherical diagram is connected and so has to lie in one of the subcomplexes.

The following result is analogous to Proposition 2.2:

Proposition 2.4. If $p: \tilde{X} \rightarrow X$ is a covering space and $\tilde{X}$ is $D R$ then $X$ is $D R$.

Proof: As in the proof of Proposition 2.2, any spherical diagram $\phi: T \rightarrow X$ lifts to a spherical diagram $\alpha: T \rightarrow \widetilde{X}$, ie $\phi$ factors as $p \circ \alpha$. Now $\widetilde{X}$ is DR. Thus $\alpha$ has a pair of opposite faces. But then the same pair of faces are opposite for $\phi$. It follows that $X$ is DR.

In [4], Gersten shows that if $\widetilde{X} \rightarrow X$ is a branched cover, branched over the zeroskeleton, and if $X$ is DR, then $\widetilde{X}$ is DR. Combining this with our previous result yields the following:

Corollary 2.5. If $p: \widetilde{X} \rightarrow X$ is a covering space then $\tilde{X}$ is $D R$ if and only if $X$ is $D R$.

Our approach to showing that a complex is Kervaire or DR is to express its universal covering space as an increasing union of subcomplexes, each of which is Kervaire or DR. Formalising this yields the following:

Lemma 2.6. Suppose a non-compact combinatorial two-complex $X$ is the direct 
limit of a family of combinatorial two-complexes $X_{\alpha}$ (where the maps are all combinatorial).

(1) If each $X_{\alpha}$ is Kervaire then $X$ is Kervaire.

(2) If each $X_{\alpha}$ is $D R$ then $X$ is $D R$.

Proof: Let $\phi: T \rightarrow X$ be a spherical diagram over $X$. Since $T$ is compact, $\phi$ factors as $g_{\alpha} \circ \phi_{\alpha}$, for some $\alpha$, where $\phi_{\alpha}: T \rightarrow X_{\alpha}$ is a spherical diagram and $g_{\alpha}: X_{\alpha} \rightarrow X$. If $X_{\alpha}$ is Kervaire then $\phi_{\alpha}$ has redundant links. Hence, by Lemma 2.1, $\phi$ has redundant links. It follows that $X$ is Kervaire. If $X_{\alpha}$ is DR then, by the above comments, $\phi$ has a pair of opposite faces. It follows that $X$ is DR.

To illustrate our approach, we will give a proof that any surface $F \neq S^{2}, R P^{2}$ is DR. We start with a lemma:

LEMma 2.7. Suppose $Y$ may be obtained from $X$ by a sequence of cellular one-moves and two-moves. If $X$ is $D R$ then $Y$ is $D R$.

Proof: As noted above, a subcomplex of a DR complex is DR. Furthermore, since spherical diagrams are combinatorial maps, cellular one-moves cannot affect the property of being DR. Thus it suffices to consider the case of $Y=X \cup \Delta$ where $\Delta$ is a two-cell attached to $X$ so that it has a free edge. Now if $\phi: T \rightarrow Y$ is a spherical diagram then either its image lies in $X$ or $\phi^{-1}(\Delta)$ is a collection of opposite faces for $\phi$ (since $T$ has no faces with free edges). In either case, our result follows.

Since there are no spherical diagrams over a one-complex, we immediately obtain a corollary:

COROLlary 2.8. If the combinatorial two-complex $Y$ collapses onto a graph then it is $D R$.

Our main result is the following:

Proposition 2.9. Let $X$ be a combinatorial two-complex and $\tilde{X}$ its universal cover. Suppose $\widetilde{X}$ is the ascending union of a family of finite subcomplexes $Y_{\alpha}$.

(1) If each $Y_{\alpha}$ collapses onto a graph then $X$ is DR.

(2) If each $Y_{\alpha}$ is Kervaire then $X$ is Kervaire.

Proof: Apply Corollary 2.8, Lemma 2.6, Proposition 2.4, and Proposition 2.2.

It is now easy to see that any surface $F \neq S^{2}, R P^{2}$ is DR. We merely observe that its universal cover, the plane $\mathbb{R}^{2}$, is an increasing union of disks and apply Proposition 2.9 .

We close this section with a lemma that we will need below.

LEMma 2.10. If $Y=X / / \Gamma$ is $D R$, where $\Gamma$ is a graph, then $X$ is $D R$. 
Proof: Suppose $\phi: T \rightarrow X$ is a spherical diagram. Collapsing each component of $\phi^{-1}(\Gamma)$ to a point yields a wedge of two-spheres mapping into $Y$. As $Y$ is DR there is a pair of opposite faces, which pull back to opposite faces for $\phi$.

\section{Modifying Surfaces}

We now turn to certain modifications of surfaces that do not affect homotopy type. We aim to prove that they do not affect Kervaireness.

Let $v(t)$ be a word in the free semigroup $A$ generated by $\left\{t, t^{-1}\right\}$ We say that $v(t)$ is plus-null if the following holds:

(1) $v(t)$ freely reduces to the null word.

(2) each initial segment of $v(t)$ has nonnegative exponent sum.

There is a topological interpertation of plus-null words. Consider the universal cover $p: \mathbb{R}^{1} \rightarrow S^{1}$. Take as cell structure for $S^{1}$ the complex $\mathcal{K}\langle t \mid\rangle$. The induced cell structure on $\mathbb{R}^{1}$ can be thought of as having vertices at the integers. Orient $S^{1}$ and $\mathbb{R}^{1}$ so that the oriented edge from 0 to 1 is a lift of the one-cell $t$. Then, considering words in $A$ as paths in $S^{1}, v(t)$ is plus-null if and only if the lift, $\widetilde{v}_{0}$ starting at 0 , is a closed path contained completely in $[0,+\infty)$.

Now consider the standard presentation of the fundamental group $G$ of the orientable surface $F$ of genus $n$ :

$$
\left\langle x_{1}, y_{1}, x_{2}, y_{2}, \ldots, x_{n}, y_{n} \mid \prod_{i=1}^{n}\left[x_{i}, y_{i}\right]\right\rangle
$$

So, in particular, the two-complex associated with the presentation is the surface $F$ itself. We thus can think of the generators $x_{i}$ and $y_{i}$ as one-cells of $F$.

The insertion of plus-null words (in the free semigroup with basis $\left\{x_{i}, y_{i}\right\}^{ \pm 1}$ ) in the presentation clearly does not change the homotopy type of the associated twocomplex. We would like to be able to prove that arbitrary insertions result in Kervaire complexes. However, our method of proof does not apply to arbitrary insertions, but only to a certain type. Let us now describe that type.

Denote by $R$ the relator in the above presentation. Suppose that $w_{1}(t), w_{2}(t), \ldots$, $w_{4 n}(t)$ are words in the free semigroup generated by $\left\{t, t^{-1}\right\}$ that freely reduce to $t$. Assume that at least one of them is of one of the following special forms:

(1) $w(t)=(t \cdot a(t))$ where $a(t)$ is a plus-null word.

(2) $w(t)=\left(t \cdot a(t) \cdot t^{-1} \cdot t \cdot b(t)\right)$ where $a(t)$ and $b(t)$ are plus-null words.

Returning to our topological approach, in case (1) one has that $\tilde{w}_{0} \cap[0,1]=e$ where $e$ is the reduced path from 0 to 1 . In case (2) one has $\tilde{w}_{0} \cap[0,1]=e \cdot e^{-1} \cdot e$. This latter path is, after identifying the vertices 0 and 1 , the attaching map for the dunce hat. 
We say that the word

$$
S=\prod_{i=1}^{n}\left(w_{4 i-3}\left(x_{i}\right) \cdot w_{4 i-2}\left(y_{i}\right) \cdot\left(w_{4 i-1}\left(x_{i}\right)\right)^{-1} \cdot\left(w_{4 i}\left(y_{i}\right)\right)^{-1}\right)
$$

is obtained from $R$ by an admissible modification (where $w_{j}(z)$ is obtained by substitution). Observe that $S$ freely reduces to $R$. Say that the modification is of type $t$ if the special form (1) holds, of type $t t^{-1} t$ if (2) holds. Let $F_{S}$ be the two-complex

$$
\mathcal{K}\left\langle x_{1}, y_{1}, x_{2}, y_{2}, \ldots, x_{n}, y_{n} \mid S\right\rangle
$$

With this terminology, our result becomes the following:

Proposition 3.1. Suppose, as above, $R=\prod_{i=1}^{n}\left[x_{i}, y_{i}\right]$. Assume $S$ is obtained from $R$ by an admissible modification.

(1) If the modification is of type $t$ then $F_{S}$ is $D R$ (and hence Kervaire).

(2) If the modification is of type $t t^{-1} t$ then $F_{S}$ is Kervaire.

Proof: We will explicitly examine the universal cover of $F_{S}$ and apply Proposition 2.9. We start by observing that the universal covering space $\widetilde{F}$ of $F$ is the plane $\mathbb{R}^{2}$. Denote the coordinates of $\mathbb{R}^{2}$ by $\mathbf{x}$ and $\mathbf{y}$. There is an induced cell structure on $\widetilde{F}=\mathbb{R}^{2}$. The zero-cells of $\widetilde{F}$ correspond to elements of $G$. The one-cells of $\tilde{F}$ are lifts of the one-cells $x_{i}$ 's and $y_{i}$ 's. (We won't distinguish between one-cells and generators from the presentation.) Assume things are arranged so that, for $i=1,2$, a lift of the one-cell $x_{i}$ is parallel to the $\mathbf{x}$-axis and in the positive $\mathbf{x}$-direction. Similarily assume that a lift the one-cell $y_{i}$ is parallel to the $y$-axis and in the positive $\mathbf{y}$-direction. The two-cells are lifts of the two-cell corresponding to $R$. Thus they are attached according to lifts of $R$. $\tilde{F}_{S}$, the universal covering space of $F_{S}$, is gotten from $\tilde{F}$ by merely changing the attaching maps to lifts of the word $S$. We need to understand exactly how to change the lifts of $R$ into lifts of $S$.

We will consider first the case $n=1$. Thus

$$
S=\left(w_{1}(x) \cdot w_{2}(y) \cdot\left(w_{3}(x)\right)^{-1} \cdot\left(w_{4}(y)\right)^{-1}\right) .
$$

Assume that $w_{2}(y)=(y \cdot a(y))$ where $a(y)$ is a plus-null word. Now $\tilde{F}$ is tessellated by rectangles with corners at the integral lattice points. We have oriented things so that the attaching maps traverse the boundary of the rectangles in a counter-clockwise direction. Take as basepoint the lower left-hand vertex. Consider the two-cell with lower left-hand vertex being the point $(j, k)$. In $\widetilde{F}_{S}$ there is a corresponding two-cell. Denote it by $S_{(j, k)}$. Its attaching map can be described as a product of four subpaths 
corresponding to lifts of the four subwords $w_{1}(x), w_{2}(y),\left(w_{3}(x)\right)^{-1}$, and $\left(w_{4}(y)\right)^{-1}$. The first subpath starts at the point $(j, k)$ and ends at the point $(j+1, k)$ remaining entirely on the line $y=k$. So in particular, its intersection with the line $x=j+1$ is only a point (namely the point $(j+1, k)$ ). In fact, one easily sees that the only part of the path of the attaching map intersecting the line $x=j+1$ in more than a point is the subpath corresponding to the lift of the subword $w_{2}(y)=(y \cdot a(y))$.

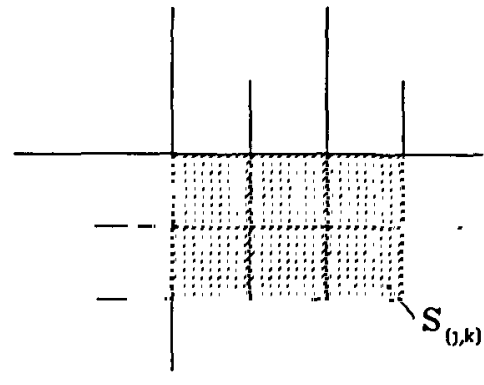

Figure 3.1

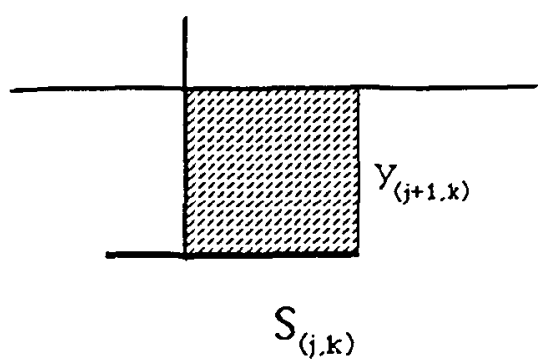

Figure 3.2

Now let $K$ be a finite subcomplex of $\widetilde{F}_{S}$. Choose $(j, k)$ so that $K$ contains the two-cell $S_{(j, k)}$ but no cell $S_{(n, m)}$ with $m \leqslant k$ and $n \neq j$. See Figure 3.1. It is then immediate from the topological interpretation of a plus-null word that the edge

$$
\mathbf{y}_{(j+1, k)}=\{(j+1, b) \mid k \leq b \leq k+1\}
$$

is free in $K$ - that is, is not in any other two-cell of $K$. See Figure 3.2 where the attaching map of $S_{(j, k)}$ is pictured. Hence, we can collapse across $S_{(j, k)}$ and so, by induction on the number of two-cells of $K$, it follows that $K$ collapses onto a graph, and thus, by Proposition 2.9, $F_{S}$ is DR.

Now assume that

$$
w_{2}(y)=\left(y \cdot a(y) \cdot y^{-1} \cdot y \cdot b(y)\right)
$$

where $a(y)$ and $b(y)$ are plus-null words. Let $K$ and $(j, k)$ be as above. Set

$$
L=\left(K \backslash S_{(j, k)}\right) \cup\left(K^{(1)} \backslash \mathbf{y}_{(j+1, k)}\right) .
$$

Using the topological interpretation we see that we have a combinatorial extension (in the sense of $[1,3]) L \rightarrow K \rightarrow X$ where $X$ is the Dunce Hat. See Figure 3.3 where the attaching map of $S_{(j, k)}$ is pictured.

Now the Dunce Hat is known to be Kervaire and by induction on the number of two-cells $L$ is Kervaire. Since combinatorial extensions preserve the Kervaire property 


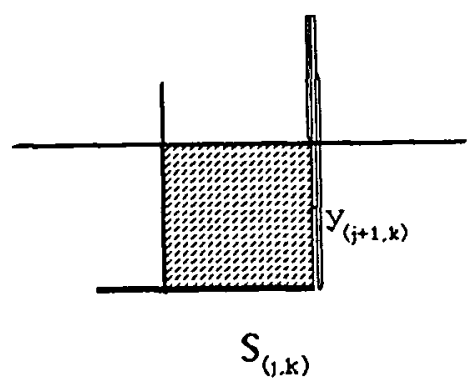

Figure 3.3

[B, Proposition 3.4], it follows that $K$ is Kervaire. And by Proposition 2.9 we have that $F_{S}$ is Kervaire.

If the word of special form is $w_{1}(x)$ then a similar argument applies to obtain our result. In the case of $w_{3}$ or $w_{4}$ we change the orientation and proceed as above. This handles the case $n=1$.

If $n \geq 2$ then only the tesselation of the plane changes. The argument is essentially the same. Alternately one can use extensions to reduce to the case of $n=1$. We assume that the word of special form is one of $w_{1}, \ldots, w_{4}$. Let $\Gamma$ be the one-complex consisting of the edges $x_{2}, y_{2}, \ldots, x_{n}, y_{n}$. Then $F_{T}=F_{S} / / \Gamma$ is DR (respectively Kervaire) by the initial case of $n=1$. Applying Lemma 2.10 (respectively [1, Proposition 3.4] as above) yields the desired result for $\boldsymbol{F}_{S}$.

This result gives us a method for generating examples of DR and Kervaire complexes. For instance, the two-complex mentioned above in the introduction,

$$
\mathcal{K}\left\langle x, y \mid x^{2} x^{-1} y^{n+1} y^{-n} x^{k} x^{-k-1} y^{-m-1} y^{m}\right\rangle,
$$

is thus seen to be DR and hence Kervaire.

Things are somewhat more complicated for modifications of non-orientable surfaces. The problem is that, in the standard presentation, some subwords are powers of generators. We will deal with this in the next section when we generalise our ideas to one-relator extensions.

\section{ONE-RELATOR EXTENSIONS}

Let $X$ be a combinatorial two-complex. We say that a combinatorial two-complex $Y$ is a one-relator extension of $X$ if the following holds:

(1) $X \subset Y$,

(2) $Y \backslash X=\{\varepsilon, \Delta\}$ where $\varepsilon$ is a one-cell and $\Delta$ is a two-cell,

(3) the attaching map, $\partial \Delta$, for $\Delta$ is cyclically reduced in the free product structure of $\pi_{1}(X \cup \varepsilon)$,

(4) the attaching map $\partial \Delta$ strictly involves $\varepsilon$, in the sense that it is not homotopic in $X \cup\{\varepsilon\}$ to a map into $X$. 
Note that this is almost equivalent, in the terminology of Howie, to saying that the pair $(Y, X)$ is an elementary reduction, the only difference being that we do not assume the two-cells of $X$ are all attached according to cyclically reduced words (see [6]).

We say that $Y$ is a one-relator extension, by a non-power, of $X$ if the attaching map $\partial \Delta$ is not, up to homotopy in $X \cup \varepsilon$, of the form $w^{n}$ for some edgepath $w$ and some integer $n \geqslant 2$.

We say that a two-complex $Z$ is staggered on one side if given any finite subcomplex $U \subset Z$ there exists an integer $n \geqslant 0$ and subcomplexes $U_{i}$ for $i=0,1, \ldots, n$ with

(1) $U_{0} \subset Z^{(1)}$

(2) $U_{i}$ is a one-relator extension of $U_{i-1}$ for each $i=1,2, \ldots, n$,

(3) $U=U_{n}$.

Howie, in [6], also calls such a complex $Z$ reducible. Further, we will say that $Z$ is staggered on one-side without proper powers if each $U_{i}$ is a one-relator extension of $U_{i-1}$, by a non-power.

There is a stronger notion of staggering. Let us recall it (see [3]). A two-complex $X$ is staggered (sometimes called staggered on both sides) if the two-cells are attached according to cyclically reduced words, and there are linear orderings of the one-cells and the two-cells of $X$, both denoted by $\prec$, so that whenever $\alpha \prec \beta$ are two-cells we have

$$
\min \{e \mid e \in \partial \alpha\} \prec \min \{e \mid e \in \partial \beta\} \quad \text { and } \quad \max \{e \mid e \in \partial \alpha\} \prec \max \{e \mid e \in \partial \beta\}
$$

where the min and max are defined via $\prec$.

In Theorem 5.5 of [3], Gersten proves that a staggered two-complex satisfies the Reciprocity Law and is hence Kervaire. We will prove a slightly sharper version in the absence of proper powers.

Note that if $Z$ is a two-complex then $Z^{\prime}=Z / Z^{(0)}$ is DR if and only if $Z$ is DR. This is true because any spherical diagram over $Z^{\prime}$ pulls back under the quotient map $Z \rightarrow Z^{\prime}$ to a spherical diagram over $Z$. This simplifies our proofs. We start with the following result (Recall that a group is said to be locally indicable if each finitely generated subgroup maps onto the integers):

Proposition 4.1. Suppose $Y$ is a one-relator extension, by a non-power, of a two-complex $X$. Assume that $X$ has a single vertex and that $\pi_{1}(X)$ is locally indicable.

(1) If $X$ is $D R$ then $Y$ is $D R$.

(2) If the universal cover $\tilde{X}$ of $X$ is Kervaire then $Y$ is Kervaire.

Proof: As above, let $Y \backslash X=\{\varepsilon, \Delta\}$ where $\varepsilon$ is a one-cell and $\Delta$ is a two-cell.

Suppose that $X$ is DR. Let $p: \tilde{Y} \rightarrow Y$ be the universal cover. We will show that $\tilde{Y}$ is DR. By Proposition 2.4, this suffices. And by Lemma 2.6, we need only see that every finite subcomplex of $\widetilde{Y}$ is DR. 
So let $K$ be a finite subcomplex of $\tilde{Y}$. We use induction on $n_{K}=\operatorname{card}\left\{K^{(2)} \backslash\right.$ $\left.p^{-1}(X)^{(2)}\right\}$. If $n_{K}=0$ then $K=L \cup \Gamma$ where $L$ is a possibly disconnected subset of $p^{-1}(X)$ and $\Gamma \subset \tilde{Y}^{(1)}$. Now $p^{-1}(X)$ is a disjoint union of covers of $X$. Each cover of $X$ is DR, by Corollary 2.5. Since a disjoint union of DR complexes is DR, and since a subcomplex of a DR complex is DR it follows that $L$ is DR. Further any spherical diagram over $K$ has image contained in $L$ since $\Gamma$ is a graph. Thus such a spherical diagram has to have a pair of opposite faces and it follows that $K$ is DR in the the case of $n_{K}=0$.

So assume the result is true for finite subcomplexes $L \subset \tilde{Y}$ with $n_{L}<n_{K}$. We will show that $K$ collapses onto a such a subcomplex.

By [6, Theorem 4.2], $\pi_{1}(Y)$ is locally indicable. And by [2, Corollary 2], $\pi_{1}(Y)$ is left-orderable. So let $\prec$ be a linear ordering on $\pi_{1}(Y)$ that is preserved by left multiplication (Note - in [2] they discuss right-orderings; of course any right-orderable group is left orderable, apply the involution $g \mapsto g^{-1}$ ).

Take the natural action of $\pi_{1}(Y)$ on $\tilde{Y}$ to be on the left. Denote the action by $\star$. Thus the zero-cells of $\widetilde{Y}$ are in one-to-one correspondence with $\pi_{1}(Y)$ and the one-cells and two-cells of $\tilde{Y}$ correspond to $g \star e$ and $g \star \alpha$ where $g \in \pi_{1}(Y), e$ is a one-cell and $\alpha$ is a two-cell of $Y$. The attaching map for the two-cell $g \star \alpha$ is somewhat complicated. Let $e \mapsto[e]$ denote the natural map $Y^{(1)} \rightarrow \pi_{1}(Y)$ (recall that $Y$ has a single two-cell). Then the attaching map is described as follows:

$$
\begin{gathered}
\partial(g \star \alpha)=\left(g \star a_{1}\right)\left(\left(g\left[a_{1}\right]\right) \star a_{2}\right) \cdots\left(\left(g\left[a_{1} a_{2} \ldots a_{i}\right]\right) \star a_{i+1}\right) \cdots\left(g\left[a_{1} \ldots a_{m-1}\right] \star a_{m}\right) \\
\text { where } \partial \alpha=a_{1} a_{2} \ldots a_{m} .
\end{gathered}
$$

Let $1 \star \partial \Delta=b_{1} b_{2} \ldots b_{k}$. We claim that the one cells

$$
b_{1},\left[b_{1}\right] \star b_{2}, \ldots,\left[b_{1} b_{2} \ldots b_{i}\right] \star b_{i+1}, \ldots,\left[b_{1} \ldots b_{k-1}\right] \star b_{k}
$$

are all distinct. This is true as otherwise we could conclude that some subword $\left[b_{i} \ldots b_{j}\right]=1$ in $\pi_{1}(Y)$ contradicting [6, Corollary 3.4]. Further it follows that the edges in the boundary of $g \star \Delta$ are all distinct.

Pick $i$ so that $\left[b_{1} \ldots b_{i}\right]$ is maximal under $\prec$ among those $i$ with $b_{i+1}=\varepsilon$ or $b_{i+1}=\bar{\varepsilon}$. Since the edges are distinct this is a strict maximum. Call this index $i_{\text {max }}$. As $\pi_{1}(Y)$ is left ordered, it follows that $g\left[b_{1} \ldots b_{i_{\max }}\right]$ is a strict maximum under $\prec$ among $g\left[b_{1} \ldots b_{i}\right]$ with $b_{i+1}=\varepsilon$ or $b_{i+1}=\bar{\varepsilon}$.

Choose $g \in \pi_{1}(Y)$ which is maximal under $\prec$ subject to the condition that $g \star e \subset \partial(h \star \Delta)$, where $e=\varepsilon$ or $e=\bar{\varepsilon}$, for some $h \star \Delta \subset K$. By the above, we know that $g=h\left[b_{1} \ldots b_{i_{\text {max }}}\right]$. We claim that $g \star e$ is a free edge in $K$. If not then there are elements $h_{1}, h_{2} \in \pi_{1}(Y)$ such that $h_{j} \star \Delta \subset K$ and $g \star e \subset \partial\left(h_{j} \star \Delta\right)$ for $j=1,2$. By 
our choice of $g$ we must have $g=h_{j}\left[b_{1} \ldots b_{i_{\max }}\right]$ for $j=1,2$. It follows that $h_{1}=h_{2}$ and thus $g \star e$ is a free edge in $K$. Collapsing across this edge reduces $n_{K}$ and by induction, using Lemma $2.7, K$ is DR. And hence $Y$ is DR.

Now suppose that the universal cover $\widetilde{X}$ of $X$ is Kervaire. We will show that the universal cover $\tilde{Y}$ of $Y$ is Kervaire. By Proposition 2.2 this suffices. The proof is identical to that above, except that we use [1, Proposition 4.3] in place of our Lemma 2.7 , and except for the initial case of induction, that is, when $n_{K}=0$. So suppose $K=L \cup \Gamma$ where $L \subset p^{-1}(X)$ and $\Gamma \subset \tilde{Y}^{(1)}$ is a graph. As a spherical diagram over $K$ has image in $L$, it suffices, by Proposition 1.1 , to show that $L$ is Kervaire. Also, since a disjoint union of Kervaire complexes is Kervaire, and since a subcomplex of a Kervaire complex is Kervaire, it suffices to see that each component $C$ of $p^{-1}(X)$ is Kervaire. By the Freiheitssatz for locally indicable groups (see [5, Theorem 4.3]), it follows that the inclusion $X \subset Y$ induces a injection on fundamental group level. But then $p\lceil C: C \rightarrow X$ is the universal cover, which by assumption is Kervaire.

Since one-complexes are DR, we immediately get the following corollary about staggered complexes.

CoROLLARY 4.2. If $Z$ is a two-complex that is staggered on one side without proper powers then $Z$ is $D R$.

We now address modifications of one-relator extensions. We will change the attaching map of the extra two-cell while preserving DR or Kervaireness. Initially, the attaching map is a cyclically reduced word in the edges of $Y$. We will restrict ourselves to a special type of extra two-cell. We need a few definitions.

Let $W$ be a cyclically reduced word in the free group on $\varepsilon, e_{1}, e_{2}, \ldots, e_{l}$. Consider $W$ as a cyclic word. A full piece will be a subword $w$ of $W$ that involves only one generator (or its inverse) and is maximal with respect to this property. So for example the full pieces of $\varepsilon e^{2} f^{-3} e \varepsilon$ are $e^{2}, f^{-3}, e$, and $\varepsilon^{2}$. We will refer to a word such as $e^{2}$ as a full $e$-piece, a word such as $f^{-3}$ as a full $f$-piece, and so on. So each such word $W$, after perhaps a cyclic conjugation can be written as a product of pieces

$$
W=W_{1}\left(f_{1}\right) \cdot W_{2}\left(f_{2}\right) \cdots W_{p}\left(f_{p}\right)
$$

where $W_{i}\left(f_{i}\right)$ is a full $f_{i}$-piece (so it is of the form $f_{i}^{m}$ ). We will replace each piece $W_{i}$ with a possibly unreduced word that freely reduces to $W_{i}$. We will assume that $W$ has only one full $\varepsilon$-piece, say $W_{1}$.

View words $w(\varepsilon)$ in the free semigroup $A$ generated by $\left\{\varepsilon, \varepsilon^{-1}\right\}$ as circuits in the one-complex $S^{1}=\mathcal{K}\langle\varepsilon \mid\rangle$. Consider the universal cover $\mathbb{R}$ of $S^{1}$. The vertices of $\mathbb{R}$ correspond to the integers. Orient things so that the edge from $i$ to $i+1$ is a lift of the one-cell $\varepsilon$. Denote it by $\varepsilon_{i}$. As in Section 3, let $\tilde{w}_{0}$ be the lift of $w(\varepsilon)$ to $\mathbb{R}$ that starts 
at the vertex 0 . Consider the word $\varepsilon^{n}$ where $n$ is positive. An admissible replacement of $\varepsilon^{n}$ is a word $w(\varepsilon)$ in $A$ that freely reduces to $\varepsilon^{n}$, such that $\widetilde{w}_{0}$ only involves $\varepsilon_{i}$ for $i \geqslant 0$ and one of the following holds:

(1) $\tilde{w}_{0} \cap\left\{\varepsilon_{0}\right\}=\varepsilon_{0}$

(2) $\tilde{w}_{0} \cap\left\{\varepsilon_{0}\right\}=\varepsilon_{0} \cdot \varepsilon_{0}^{-1} \cdot \varepsilon_{0}$

In the first case we call $u$ a replacement of type $t$, in the latter case a replacement of type $t t^{-1} t$. An admissible replacement of $\varepsilon^{m}$, where $m$ is negative, is simply $w^{-1}$ where $w$ is an admissible replacement of $\varepsilon^{|m|}$.

So given a cyclically reduced word $W$ in the free group on $\varepsilon, e_{1}, e_{2}, \ldots, e_{l}$, we write

$$
W=W_{1}(\varepsilon) \cdot W_{2}\left(f_{2}\right) \cdots W_{p}\left(f_{p}\right)
$$

where, for each $i \geqslant 2, f_{i} \in\left\{\varepsilon, e_{1}, e_{2}, \ldots, e_{l}\right\}$ and $W_{i}\left(f_{i}\right)$ is a full $f_{i}$-piece. Assume that we have that $U_{1}(\varepsilon)$ is an admissible replacement of $W_{1}(\varepsilon)$. And assume that for $i \geqslant 2$ $U_{i}\left(f_{i}\right)$ freely reduces to $W_{i}(t)$. Then we will call the word

$$
U=U_{1}(\varepsilon) \cdot U\left(f_{2}\right) \cdots U\left(f_{p}\right)
$$

an admissible modification of $W$. We will call $U$ a modification of type $t$ if the subword $U_{1}$ is of type $t$. Otherwise, $U$ is of type $t t^{-1} t$.

Suppose that $Y$ is a one-relator extension of $X$ by a non-power with extra one-cell $\varepsilon$ and two-cell $\Delta$. The attaching map for $\Delta$ is represented by a cyclically reduced word in the free group on the edges of $Y$. Assume that the attaching map has only one full $\varepsilon$-piece. We say that $Z$ is obtained from $Y$ by an admissible modification if

$$
Z=\left\{\Delta^{\prime}\right\} \cup(Y \backslash\{\Delta\})
$$

where the attaching map $U$ for $\Delta^{\prime}$ is an admissible modification of the attaching map $W$ for $\Delta$. We say that the modification is of type $t$ or $t t^{-1} t$ according to which type $U$ is. We adapt the proof of Proposition 4.1. Because we are assuming that there is only one full $\varepsilon$-piece we can omit the local indicabillity requirement.

Proposition 4.3. Suppose $Y$ is a one-relator extension, by a non-power, of a two-complex $X$ with a single full $\varepsilon$-piece. Assume that $X$ has a single vertex. Let $Z$ be an admissible modification of $Y$.

(1) If $X$ is DR and $Z$ is of type $t$ then $Z$ is DR.

(2) If the universal cover $\widetilde{X}$ of $X$ is Kervaire, and $Z$ is of either type, then $Z$ is Kervaire.

Proof: The proof is a simple modification of the proof of Proposition 4.1. It suffices, as it did above, to prove by induction on $n_{K}=\operatorname{card}\left\{K^{(2)} \backslash p^{(-1)}(X)^{(2)}\right\}$ that 
each finite subcomplex $K$ of $\tilde{Z}$ is DR in case 1 and Kervaire in case 2. The argument given in the proof of Proposition 4.1 applies if $n_{K}=0$. So assume the result for subcomplexes $L \subset \tilde{Z}$ with $n_{L}<n_{K}$.

Take a component $\ell$ of $p^{-1}(\varepsilon)$ that has intersection with $K$ larger than a collection of vertices. This component can be identified with the real line with vertices at integral points. Denote the edges of $\ell$ by $e_{i}=[i, i+1]$ where $i \in \mathbb{Z}$. We assume that things are orientated so that $\varepsilon$ lifts to some $e_{i}$ (as opposed to some $e_{i}^{-1}$ ). Further assume that the full $\varepsilon$-piece of the extra two cell of $Y$ has positive exponent (the argument for negative exponent is similar; merely reverse the orientation of $\ell$ ). Since $K$ is finite, there is a left-most edge, $e_{i}$, in $\ell \cap K$. And there is a unique two-cell $\Delta$ in $K$ with $e_{i}$ lying in $\partial \Delta$ (see Figure 4.1). Since the modification is admissible, either $e_{i}$ is free in $K$ or we get an extension of some $L$, with $n_{L}<n_{K}$, by the Dunce Hat. In any case, as in Proposition 4.1 we obtain the desired result.

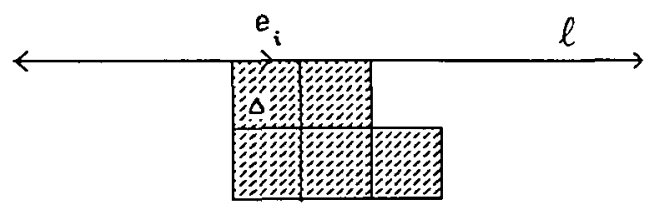

$\mathrm{K}$

Figure 4.1

As mentioned in Section 3, the preceding handles modifications of non-orientable surfaces (other than the projective plane). Also, it is clear that we can enlarge the class of admissible modifications once we know more equations in one unknown, over groups, that can be solved.

\section{A Conjecture}

We close with the following conjecture (see Proposition 2.9):

Conjecture. Let $X$ be a combinatorial two-complex and $\tilde{X}$ its universal cover. Suppose that $X$ is DR. Then $\tilde{X}$ is the ascending union of a family of finite subcomplexes, each of which collapses onto a graph.

\section{REFERENCES}

[1] S.G. Brick, 'Normal-convexity and equations over groups', Invent. math. 94 (1988), 81-104. 
[2] R.G. Burns and V.W.D. Hale, 'A note on group rings of certain torsion-free groups', Canad. Math. Bull. 15 (1972), 441-445.

[3] S.M. Gersten, 'Reducible diagrams and equations over groups', in Essays in group theory (MSRI Publ., Vol. 8), pp. 15-74 (Springer-Verlag, Berlin, Heidelberg, New York, 1987).

[4] S.M. Gersten, 'Branched coverings of 2-complexes and diagrammatic asphericity', Trans. Amer. Math. Soc. 303 (1987), 689-706.

[5] J. Howie, 'On pairs of 2-complexes and systems of equations over groups', J. Reine Angew. Math. 324 (1981), 165-174.

[6] J. Howie, 'On locally indicable groups', Math. Z. 180 (1982), 445-461.

[7] J. Howie, 'Spherical diagrams and equations over groups', Proc. Camb. Philos. Soc. 96 (1984), 255-268.

Department of Mathematics

California State University at Fresno

Fresno, CA 93740

United States of America 